\title{
IMPORTÂNCIA DA DERMATOSCOPIA EM LESÃO INCIPIENTE DE LINFANGIOMA CIRCUNSCRITO NEVIFORME EM CRIANÇA
}

\section{ARTIGO ORIGINAL}

ZÁU, Aline Sales Mendes ${ }^{1}$, ROCHA, Ana Paula Coelho ${ }^{2}$, SANTOS, Luciana Mendes $\operatorname{dos}^{3}$

ZÁU, Aline Sales Mendes. ROCHA, Ana Paula Coelho. SANTOS, Luciana Mendes dos. Importância da dermatoscopia em lesão incipiente de linfangioma circunscrito neviforme em criança. Revista Científica Multidisciplinar Núcleo do Conhecimento. Ano. 07, Ed. 01, Vol. 06, pp. 133-139. Janeiro de 2022. ISSN: 24480959, Link de acesso: https://www.nucleodoconhecimento.com.br/saude/lesaoincipiente

\section{RESUMO}

Contexto: O Linfangioma Circunscrito Neviforme (LCN) é uma malformação linfática benigna rara que corresponde a um hamartoma e ocorre nos primeiros anos de vida. Caracteriza-se clinicamente por múltiplas micropápulas vesiculosas de conteúdo claro e/ou serohemático e possui diversos diagnósticos diferenciais. Problema: Diante de uma doença rara, com clínica semelhante à numerosas dermatoses, a dermatoscopia pode ser uma ferramenta importante a fim de nortear e aprimorar o diagnóstico. Objetivo: Relata-se o caso de uma menina, 8 anos, que há 5 anos estava sendo tratada como Molusco Contagioso por apresentar micropápulas normocrômicas vesiculosas agrupadas e assintomáticas no abdome. A análise e interpretação dos

1 Graduação em medicina, residente do terceiro ano do serviço de dermatologia. ORCID: 0000-0002-6561-376X

2 Graduação em medicina, residente do terceiro ano do serviço de dermatologia. ORCID: 0000-0001-9895-706X

${ }^{3}$ Orientador. ORCID: 0000-0002-3991-6732

RC: 106004

Disponível em: https://www.nucleodoconhecimento.com.br/saude/lesao-incipiente 
padrões dermatoscópicos das lesões permitiram a hipótese de LCN que foi confirmado pelo exame histopatológico. Metodologia: Realizou-se anamnese completa, exame físico dermatológico, incluindo avaliação dermatoscópica e biópsia incisional da paciente em questão, constatando-se tratar de um caso de LCN. Para a construção do artigo, realizou-se, também, uma breve revisão de literatura sobre os aspectos dermatoscópicos dessa lesão. Conclusão: Conclui-se que a dermatoscopia é uma ferramenta importante para melhorar a acurácia diagnóstica de uma patologia rara como o LCN, sendo necessário conhecer seus aspectos dermatoscópicos a fim de excluir outros diagnósticos e evitar tratamentos desnecessários sem diagnóstico definitivo.

Palavras-chave: Linfangioma circunscrito, Malformação linfática, Dermatoscopia, Molusco contagioso, Micropápulas vesiculosas.

\section{INTRODUÇÃO}

O LCN é uma malformação linfática benigna rara, que corresponde a um hamartoma, conforme descrito por Domínguez (2013), clinicamente caracterizado pela presença de múltiplas micropápulas vesiculosas de conteúdo claro e/ou serohemático segundo Jucá et al. (2011), que podem coalescer, formar crostas e eventualmente ter um aspecto quase verrucoso. De acordo com Guerrero (2013) geralmente surgem na infância, e não há predileção por raça ou sexo.

Esta malformação deve ser lembrada como importante diagnóstico diferencial de molusco contagioso (MC), verruga vulgar, angiomas, angioceratomas, linfangioendoteliomas e angiossarcomas. Neste contexto, o presente artigo, tem como problemática: diante de uma doença rara, com clínica semelhante à numerosas dermatoses, a dermatoscopia pode ser uma ferramenta importante para distingui-las a fim de nortear e aprimorar a suspeita clínica e diagnóstica.

Portanto, tem como objetivo relatar o caso de uma menina, 8 anos, que há 5 anos estava sendo tratada como molusco contagioso por apresentar micropápulas

RC: 106004

Disponível em: https://www.nucleodoconhecimento.com.br/saude/lesao-incipiente 
normocrômicas vesiculosas agrupadas e assintomáticas no abdome. A análise e interpretação dos padrões dermatoscópicos das lesões permitiram a hipótese de LCN que foi confirmado pelo exame histopatológico. Realizou-se anamnese completa, exame físico dermatológico, avaliação dermatoscópica e biópsia incisional, constatando-se tratar de um caso de LCN.

\section{METODOLOGIA}

A paciente em estudo foi atendida no ambulatório do serviço de dermatologia do Hospital Universitário Getúlio Vargas de Manaus/AM com queixa de lesões cutâneas em micropápulas normocrômicas vesiculosas agrupadas e assintomáticas no abdome. Esta foi submetida a anamnese completa, exame físico dermatológico, incluindo avaliação dermatoscópica e biópsia incisional, constatando-se tratar de um caso de Linfangioma Circunscrito Neviforme.

\section{RELATO DE CASO}

Paciente 8 anos, sexo feminino, veio ao ambulatório do serviço de dermatologia do Hospital Universitário Getúlio Vargas de Manaus/AM. A paciente queixava-se de lesão cutânea discreta há 5 anos, localizada no abdome, assintomática, de crescimento insidioso em número, realizou diversos tratamentos prévios por suspeita diagnóstica de molusco contagioso sem melhora clínica. Nega prurido, acometimento em outras áreas do corpo, alteração do estado geral ou outras queixas. O exame dermatológico revelou a presença de múltiplas pápulas vesiculosas, milimétricas, cor da pele, translúcidas, com superfície lisa e brilhante, algumas vinhosas, agrupadas no abdome sobre uma base normocrômica (Fig. 1). Não foi realizado exame de imagem para analisar a existência de componente profundo. À dermatoscopia mostrou padrão lacunar múltiplo, separadas por septos claros, com conteúdo translúcido e, em menor quantidade, serohemático (Fig. 2). Os referidos achados dermatoscópicos eram compatíveis com o diagnóstico de LCN, e, como complementação dos exames para elucidação diagnóstica, foi realizado uma biópsia incisional que se confirmou pelo 
estudo histopatológico se tratar de LCN. Diante da benignidade do diagnóstico e ausência de sintomatologia clínica, optou-se por manter conduta expectante.

Figura 1: Múltiplas pápulas vesiculosas, milimétricas, cor da pele, translúcidas, de superfície lisa e brilhante, algumas vinhosas e coalescentes.

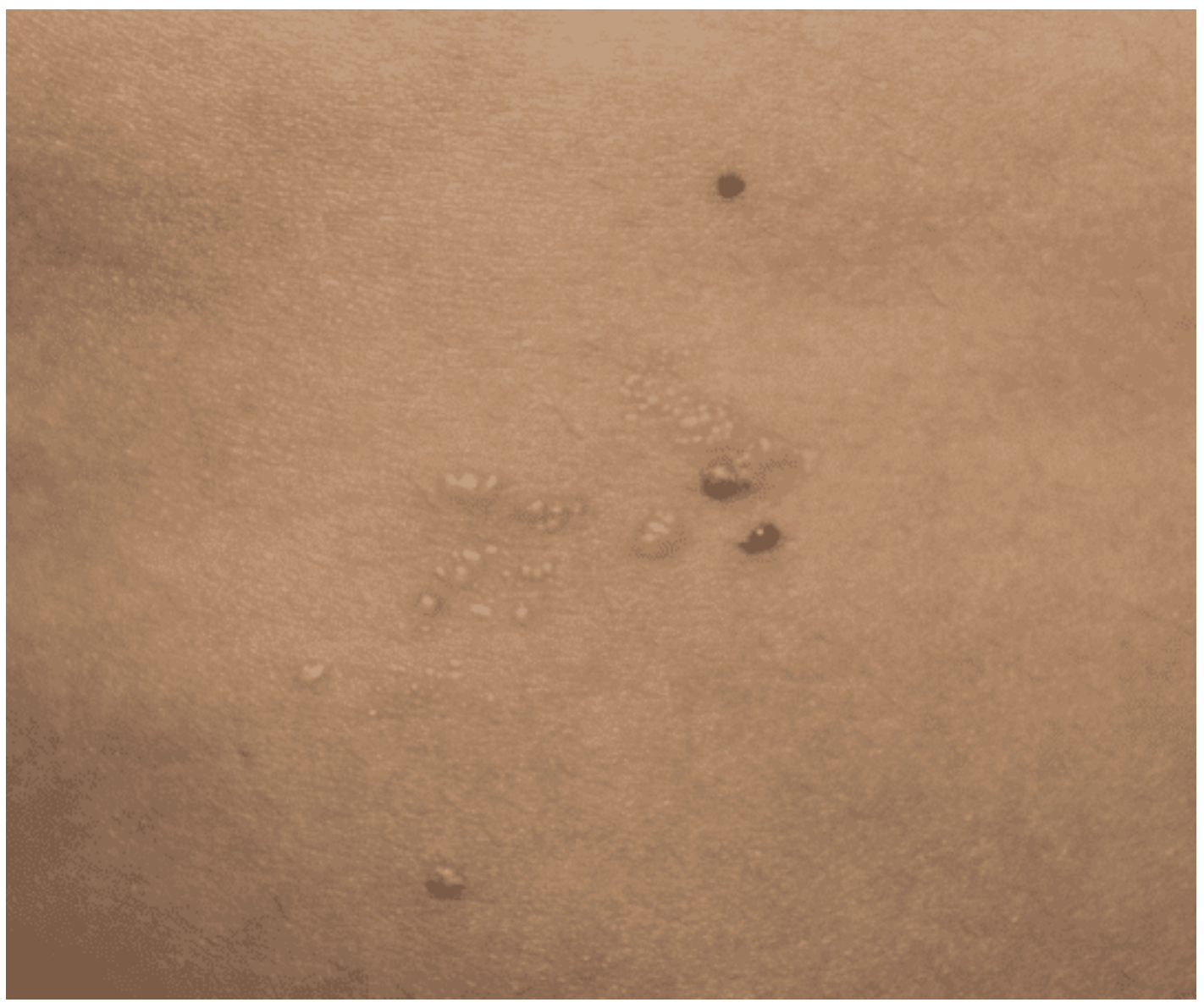

Fonte: arquivo do serviço de dermatologia HUGV, 2019.

RC: 106004

Disponível em: https://www.nucleodoconhecimento.com.br/saude/lesao-incipiente 
Figura 2: Padrão lacunar múltiplo, separadas por septos claros, com conteúdo translúcido e serohemático.

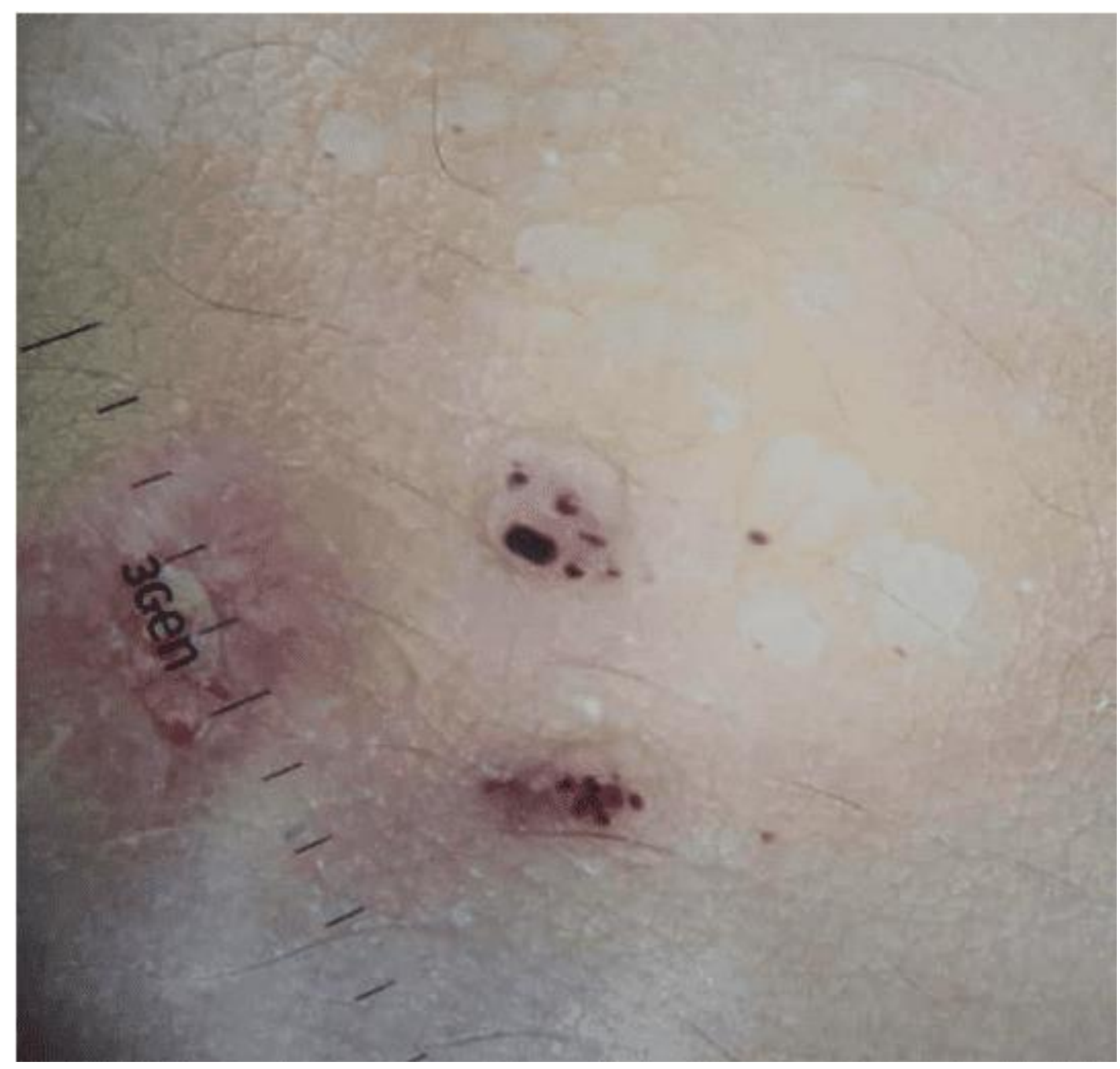

Fonte: arquivo do serviço de dermatologia HUGV, 2019.

RC: 106004

Disponível em: https://www.nucleodoconhecimento.com.br/saude/lesao-incipiente 
Figura 3: Vasos arboriformes em um padrão de coroa, com centro branco amarelado.

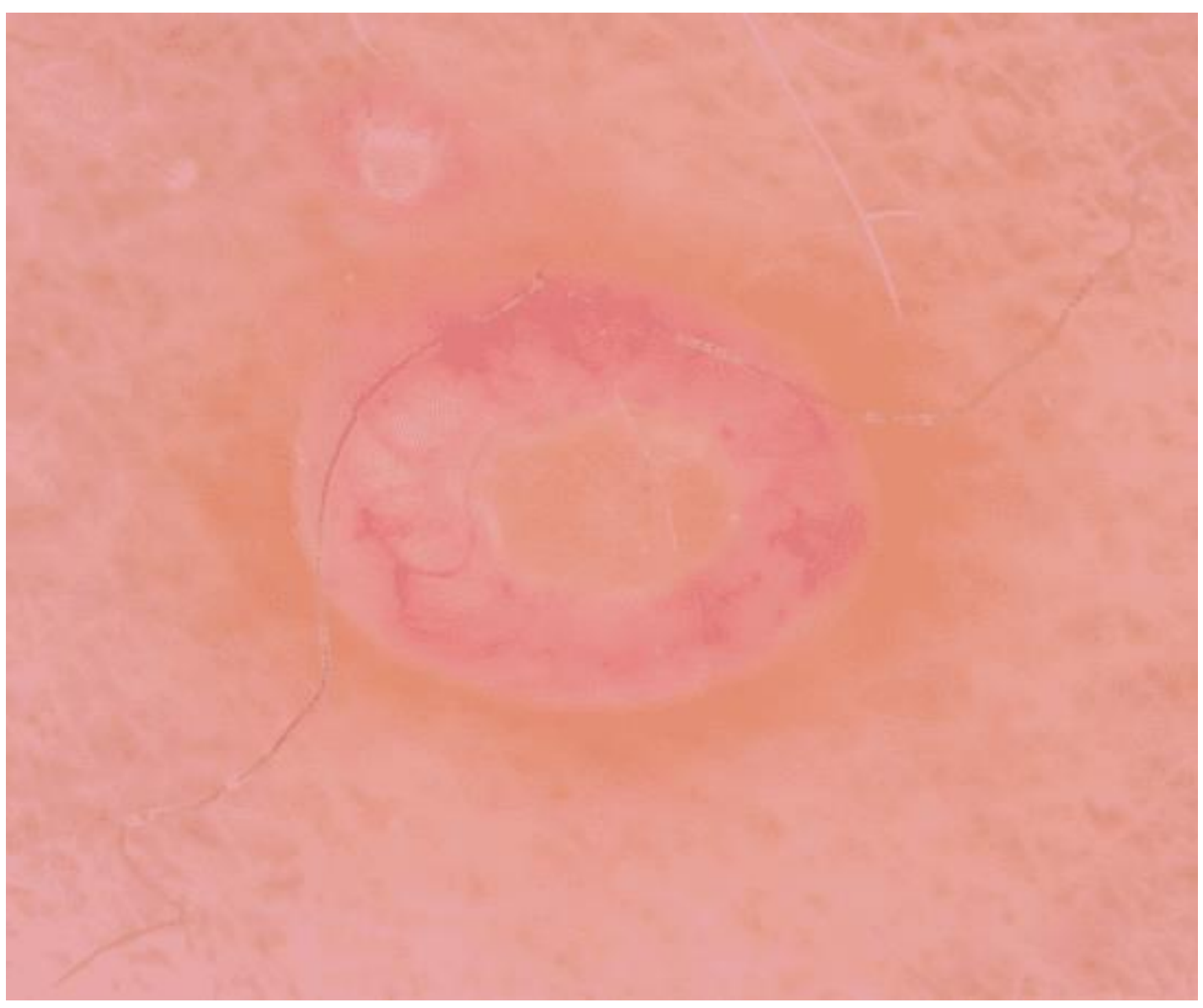

Fonte: https://dermagarrafblog.wordpress.com/2017/04/22/ficha-19-dermatoscopiaen-infecciones-e-infestaciones/

\section{DISCUSSÃO}

O LCN é uma malformação linfática microcística congênita rara e benigna de etiologia incerta. Comumente surgem entre 2-3 anos de idade como demonstra a literatura de Boon; Vikkula (2008). O pico de incidência é até os 5 anos de idade, sendo que $90 \%$ apresentam-se antes dos 2 anos, como no caso aqui apresentado. Localiza-se geralmente em região axilar, inguinal, extremidades (GONTIJO, 2004) e boca, sendo incomum sua localização na região do abdome como descrito nesta paciente. 
A hipótese de Whimster (MENCHÓN, 2004) associa sua origem ao aumento da pressão ao nível dos vasos linfáticos da pele, os quais se encontrariam conectados a cisternas linfáticas hipodérmicas, assim, justificando a drenagem ocasional de linfa. Seu acometimento profundo, especialmente em lesões bem desenvolvidas e extensas, torna a abordagem terapêutica um desafio. Existe a possibilidade de conduta expectante para lesões menores, excisão cirúrgica, ablação com laser de dióxido de carbono, crioterapia, eletrofulguração, radiofrequência, embolização, dentre outras, porém nenhuma ideal e com possibilidade de recidiva e cicatrizes inestéticas (GUERRERO, 2013).

A clínica do LCN é semelhante à dos angiomas, angioceratomas, molusco contagioso, linfangioendoteliomas, angiossarcomas, verrugas, hidrocistoma e herpes simples, e os achados dermatoscópicos são fundamentais para realizar diagnóstico diferencial. O Linfangioma apresenta dois padrões dermatoscópicos distintos, o primeiro com lagos amarelos envoltos por septo claro sem vasos sanguíneos, e outro com lagos amarelo-avermelhados ou vermelho-enegrecidos contendo sangue (MASSA, 2015). Em se tratando de molusco contagioso, patologia para qual a paciente estava sendo tratada há cerca de 5 anos, seria esperado dermatoscopicamente um centro constituído por área amorfa amarela esbranquiçada circundada por vasos arboriformes em coroa, lineares ou ramificados, que não intersectam o centro (Fig. 3), segundo Pedrosa et al. (2013). A dermatoscopia foi essencial para o diagnóstico de uma lesão crônica, porém com clínica incipiente, assim, é de fundamental importância que o especialista tenha conhecimento sobre esses achados afim de ter o LCN como possibilidade diagnóstica.

\section{CONCLUSÃO}

Baseado na literatura e na peculiaridade do relato de caso apresentado, conclui-se que o LCN é uma patologia congênita rara que possui diversos diagnósticos clínicos diferenciais. Assim, a dermatoscopia é uma ferramenta importante para melhorar a acurácia diagnóstica desta patologia, é necessário que o especialista conheça seus

RC: 106004

Disponível em: https://www.nucleodoconhecimento.com.br/saude/lesao-incipiente 
aspectos dermatoscópicos a fim de excluir outras patologias e evitar tratamentos desnecessários sem o diagnóstico definitivo.

\section{REFERÊNCIAS}

BOOM L, VIKKULA M. Vascular Malformations.In: Wolff $\mathbf{K}$, Johnson RA, editors. Fitzpatrick's Color Atlas And Synopsis Of Clinical Dermatology. Berkshire: McGraw Hill; 2008.p. 1651-66.

DOMÍNGUEZ, VG. Dermoscopia de linfangioma circumscriptum neviforme relato de caso. Revista da Sociedade Portuguesa de Dermatologia e Venereologia, v. 70, n. 3, p. 369, 13 de janeiro de 2013.

GONTIJO, Bernardo et al. Malformações vasculares. Anais Brasileiros de Dermatologia, v. 79 , n. 1, p.7-25, 2004. Disponível em: $<$ https://doi.org/10.1590/S0365-05962004000100002>. Epub 17 Jun 2004. ISSN 1806-4841. https://doi.org/10.1590/S0365-05962004000100002. Acesso em: 18 jan. 2022.

GUERRERO, María et al. Linfangioma circunscrito zosteriforme. Presentación de un caso. Medisur, Cienfuegos, v. 11, n. 2, p. 195-200, abr. 2013. Disponible en <http://scielo.sld.cu/scielo.php?script=sci_arttext\&pid=S1727897X2013000200012\&lng=es\&nrm=iso>. accedido en 19 enero 2022.

JUCÁ, Natália Braga Hortêncio et al. Linfangioma microcístico acral: diagnóstico diferencial em lesões verrucosas de extremidades. Anais Brasileiros de Dermatologia, v. $86, \quad$ n. 2 , p. 343-6, 2011. Disponível em: http://www.scielo.br/pdf/abd/v86n2/v86n2a20.pdf. Acesso em: 18 jan. 2022.

MARTÍNEZ-MENCHÓN, T et al. Lymphangioma circumscriptum: an example of Whimster's hypothesis. Pediatr Dermatol., v. 21, n. 6, p. 652-4, nov-dec 2004. DOI 10.1111/j.0736-8046.2004.21607.x. PMID: 15575849. Disponível em: https://pubmed.ncbi.nlm.nih.gov/15575849/. Acesso em: 18 jan. 2022.

RC: 106004

Disponível em: https://www.nucleodoconhecimento.com.br/saude/lesao-incipiente 
MASSA, António Fernandes et al. Cutaneous Lymphangioma circumscriptum dermoscopic features. Anais Brasileiros de Dermatologia, v. 90, n. 2, p. 262-264, 2015. DOI https://doi.org/10.1590/abd1806-4841.20153652. Disponível em: https://doi.org/10.1590/abd1806-4841.20153652. Acesso em: 18 jan. 2022.

PEDROSA, A.; MORAIS, P.; MOREIRA, C.; FERREIRA, O.; AZEVEDO, F. Dermoscopia - casos selecionados de aplicações sub explorados e revisão da literatura. Revista da Sociedade Portuguesa de Dermatologia e Venereologia, v. 71, n. 4, pág. 487-494, 6 de julho de 2014.

Enviado: Janeiro, 2022.

Aprovado: Janeiro, 2022. 